[2] Yokosawa $\mathrm{M}$, et al. T-bet over-expression regulates aryl hydrocarbon receptor-mediated $\mathrm{T}$ helper type 17 differentiation through an interferon gindependent pathway. Clin Exp Immunol 2016;188:22-35.

[3] Szabo, et al. Distinct effects of T-bet in $\mathrm{TH} 1$ lineage commitment and IFNg production in CD4 an CD8 T cells. Science. 2002;295:338-42.

Disclosure of Interests: Masaru Shimizu: None declared, Yuya Kondo: None declared, Kotona Furuyama: None declared, Masahiro Yokosawa: None declared, Hiroto Tsuboi: None declared, Isao Matsumoto: None declared, Takayuki Sumida Grant/research support from: Bristol-Myers Squibb, Speakers bureau: Bristol-Myers Squibb

DOI: 10.1136/annrheumdis-2019-eular.3675

\section{SAT0067 SECRETORY ANTIBODIES TO CITRULLINATED PEPTIDES IN PLASMA AND SALIVA FROM RHEUMATOID ARTHRITIS PATIENTS AND THEIR UNAFFECTED FAMILY MEMBERS}

Anna Svärd ${ }^{1}$, Karin Roos Ljungberg ${ }^{1,2}$, Mikael Brink ${ }^{3}$, Alf Kastbom ${ }^{2}$, Solbritt Rantapää Dahlqvist ${ }^{3} .{ }^{1}$ Center for Clinical Research Dalarna, Uppsala university, Falun, Sweden; ${ }^{2}$ 2. Department of Rheumatology and Department of Clinical and Experimental Medicine, Linköping University, Linköping, Sweden; ${ }^{3} 3$. Department of Public Health and Clinical Medicine, Rheumatology, Umeå University, Umeå, Sweden

Background: Mucosal involvement in early phases of rheumatoid arthritis (RA) pathophysiology has emerged as an attractive hypothesis, supported by several findings. Elevated levels of antibodies against citrullinated peptides/proteins (ACPA) can be found during a long pre-symptomatic period, preceding manifest arthritis. Secretory antibodies are produced at mucosal surfaces, but can also be detected in the circulation. Secretory ACPA in plasma has been demonstrated in patients with RA (1). First-degree relatives (FDRs) of patients with RA can be regarded as potential pre-RA patients or at-risk individuals. In a previous study, a higher prevalence of ACPA was found in FDRs than in healthy controls (2), with IgA-ACPA being more common than IgG-ACPA.

We hypothesized that formation of secretory ACPA is an early step in RA development, preceding the occurrence of regular non-secretory ACPA, and consequently secretory ACPA would be prevalent in a large proportion of FDRs.

Objectives: To evaluate secretory ACPA in plasma and saliva from patients with RA and FDRs.

Methods: We analyzed secretory antibodies to 2nd generation cyclic citrullinated peptides (anti-CCP) in plasma from 194 patients with RA and 191 FDRs unaffected by RA and salivary samples from 25 RA patients, 21 first-degree relatives and 11 controls.

In plasma, cutoff for secretory ACPA was set at the $99^{\text {th }}$ percentile of healthy blood donors. In saliva, a positive test was defined as a difference between optical density values for $\lg \mathrm{A}$ anti-CCP and $\lg \mathrm{A}$ anti-cyclic arginine peptide (delta OD value) $>2$ SD above the mean delta OD value of the controls.

Mann-Whitney $U$ test was used for continuous variables and Pearson Chi-square for categorical variables.

Results: Secretory ACPA occurred in $37(19.1 \%)$ of RA patients but only in $2(1 \%)$ of FDRs (table 1). Salivary IgA ACPA was found in $3 / 25$ $(12 \%)$ of patients with RA, but not in any of the 21 FDRs. $27 \%$ of FDRs were positive for regular non-secretory IgA ACPA in plasma, and out of them, only 2 individuals $(5 \%)$ were positive for secretory ACPA in plasma. Among FDRs negative for regular ACPAs, no one was positive for secretory ACPA. Secretory ACPA had the highest PPV for identifying patients, while IgG ACPA had the highest negative predictive value (table 2).

Table 1. Demographic and laboratory characteristics of the study participants

\begin{tabular}{lccc}
\hline & $\begin{array}{c}\text { FDRs } \\
(\mathbf{n}=191)\end{array}$ & $\begin{array}{c}\text { RA patients } \\
(\mathbf{n}=194)\end{array}$ & P value \\
\hline Age (years), median (IQR) & $60(26)$ & $66(18.3)$ & $<0.001$ \\
Female $\mathrm{n}(\%)$ & $111(58.1)$ & $136(70.1)$ & 0.014 \\
Shared epitope, $\mathrm{n}(\%)$ & $83(53.9)$ & $116(71.2)$ & 0.001 \\
Smoker ever, $\mathrm{n}(\%)$ & $81(47.9)$ & $109(58.0)$ & 0.057 \\
IgG ACPA+, $\mathrm{n}(\%)$ & $34(21.7)$ & $140(85.9)$ & $<0.001$ \\
IgA ACPA+, n (\%) & $42(26.8)$ & $118(72.4)$ & $<0.001$ \\
IgM ACPA+, n (\%) & $35(22.3)$ & $74(45.4)$ & $<0.001$ \\
Secretory ACPA+, n (\%) & $2(1)$ & $37(19.1)$ & $<0.001$ \\
IgM RF+, n (\%) & $22(14)$ & $121(74.2)$ & $<0.001$ \\
IgA RF+, n (\%) & $35(22.3)$ & $123(75.5)$ & $<0.001$ \\
\hline
\end{tabular}

Table 2. Performance of circulating antibodies to identify patients with RA vs first-degree relatives

\begin{tabular}{lcccc}
\hline & $\begin{array}{c}\text { Sensitivity } \\
(\%)\end{array}$ & $\begin{array}{c}\text { Specificity } \\
(\%)\end{array}$ & $\begin{array}{c}\text { PPV } \\
(\%)\end{array}$ & $\begin{array}{c}\text { NPV } \\
(\%)\end{array}$ \\
\hline $\begin{array}{l}\text { Secretory ACPA+, n } \\
\text { (\%) }\end{array}$ & 19.1 & 99.0 & 94.9 & 54.6 \\
IgG ACPA+, n (\%) & 72.2 & 82.2 & 80.5 & 74.4 \\
IgA ACPA+, n (\%) & 60.8 & 78.0 & 73.8 & 66.2 \\
IgM ACPA+, n (\%) & 38.1 & 81.7 & 67.9 & 56.5 \\
IgM RF+, n (\%) & 62.4 & 88.5 & 84.6 & 69.8 \\
IgA RF+, n (\%) & 63.4 & 81.7 & 77.8 & 68.7 \\
\hline
\end{tabular}

Conclusion: Secretory ACPA in plasma and saliva was rare in FDRs. Circulating secretory ACPA had the highest PPV of the RA-related antibodies tested to identify RA in these families. This implies that mucosal production of ACPA is not common in an at-risk population, but may be important in the transition from increased risk to actual RA disease.

\section{REFERENCES}

[1] Roos K, et al. Arthritis Res Ther. 2016;18(1):119.

[2] Young KA, et al. Arthritis Rheum. 2013;65(8):1995-2004.

Disclosure of Interests: Anna Svärd: None declared, Karin Roos Ljungberg: None declared, Mikael Brink Shareholder of: Owns shares in Bris tol-Myers Squibb Co, Alf Kastbom Consultant for: Roche and Pfizer, Employee of: Sanofi, Speakers bureau: UCB and BMS, Solbritt Rantapää Dahlqvist Consultant for: Member of the advisory board, Lipum AB, Umeå, Sweden.

DOI: 10.1136/annrheumdis-2019-eular.3477

\section{SAT0068 CIRCADIAN RHYTHMS OF IMMUNE SYSTEM IN HEALTHY INDIVIDUALS AND PATIENTS WITH RHEUMATOID ARTHRITIS}

Siska Wilantri $^{1,2}$, Cindy Strehl $^{1,2}$, Dimas Abdirama ${ }^{1,2}$, Timo Gaber $^{1,2}$, Frank Buttgereit ${ }^{1,2} .{ }^{1}$ Charité - Universitätsmedizin Berlin, Rheumatology and Clinical Immunology, Berlin, Germany; ${ }^{2}$ Deutsches Rheuma-Forschungszentrum (DRFZ), AG Buttgereit (Glucocorticoid and Bioenergetic), Berlin, Germany

Background: Chronobiological aspects play an important role in rheumatoid arthritis (RA), with major symptoms such as joint pain and stiffness follow a day-night-cycle that peak in the early morning. The circadian behavior of symptoms has been attributed to the rhythmic expression of cytokines and hormones ${ }^{1},{ }^{2}$. Animal models of autoimmune inflammation demonstrated that immune cells, which belong to the vast producers of such cytokines, circulate in a circadian manner and that the circadian rhythm of circulating immune cell is different in health and disease ${ }^{3}$. Moreover, coordinating timing of glucocorticoid therapy (chronotheraphy) results in a greater reduction of morning stiffness and pain compared with the same glucocorticoid dose taken in the morning ${ }^{4}$.

Objectives: Here, we aim to analyse the circadian rhythms of circulating immune cell populations in the periphery of healthy individuals and RA patients in order to optimize treatment strategies.

Methods: We performed a 24-hours study involving 14 eligible RA patients and 12 healthy individuals to monitor the dynamic occurrence of diverse immune cells in the periphery. A week prior to study day, the biological rhythms were synchronized by scheduled activities and food intakes. On the study day, blood was drawn every 2 hours throughout 24 hours using peripheral venous catheter. The participants were provided with regular meal, allowed to eat snacks ad libitum and carry passive activities. Blood samples were subsequently analyzed by flow cytometry using TruCount Beads to count absolute cell numbers. RNA from CD14 ${ }^{+}$ monocytes was analyzed by real-time PCR to monitor the circadian clock genes expression.

Results: We investigated major immune populations and found chronobiological differences in NK T cells, NK cells, CD8 T cells, CD4 T cells and regulatory $T$ cells (Tregs) of healthy individuals and patients with RA. In RA Tregs showed a lag phase of 5 hours, while CD8 and CD4 $\mathrm{T}$ cells showed a mild reduction of the amplitude. NK $\mathrm{T}$ cells and NK cells did not circulate in a circadian manner in healthy individuals, but they exhibited circadian pattern in RA. In addition, the GPCR data also indicated a disrupted circadian rhythm on RNA level. Among ten clock genes that were examined, REVERB $\alpha, P E R 1$, and PER3 showed altered expression in RA patients. The expression of $R E V E R B \alpha$ was suppressed by $50 \%$ and the rhythm of PER1 was diminished in RA patient. 
Furthermore, the rhythm of PER3 was present exclusively in RA patients, but not in healthy participants.

Conclusion: Taken together, we conclude that the circadian rhythms of immune cells in RA patients are different from that in healthy individuals. The alteration can be observed on both cellular and RNA level. We postulate that the alterations in circadian rhythms may contribute to disease manifestations and may have impact for optimizing diagnosis and therapy in RA. Further analysis is required to expand the data set. It is also of interest to investigate the functionality of circadian clock genes in immune cells.

\section{REFERENCES}

[1] Cutolo M, et al. Ann. Rheum. Dis. 67, 905-8 (2008).

[2] Straub RH, et al. Arthritis Rheum. 56, 399-408 (2007).

[3] Druzd D, et al. Immunity. 46(1),120-132 (2017).

[4] Buttgereit, et al. Lancet. 371: 205-14 (2008).

Acknowledgement: We thank our study team; R. Biesen, E. Wiebe, K-N. Zeiner, D. Freier, M. Jakstadt, L. Ehlers, A. Damerau, A. Lang, M. Pfeiffenberger, G. May, P-L. Krauß, M. Ibach, F. Heinsohn, A. Brinkman, Y. Chen and J. Pienczykowski for their contribution.

Disclosure of Interests: None declared

DOI: 10.1136/annrheumdis-2019-eular.1352

\section{SAT0069 PROTECTIVE EFFECT OF PROTOCATECHUIC ACID RICH FRACTION OF TRIANTHEMA PORTULACASTRUM AGAINST COLLAGEN INDUCED RHEUMATOID ARTHRITIS VIA GUT MICROBIOTA MODULATION}

\section{Ekta Yadav. SHUATS, Allahabad, India}

Background: Rheumatoid arthritis, a chronic inflammatory joint disease affects people all over the world. Trianthema portulacastrum (TP) has been traditionally utilized for the treatment of rheumatism.

Objectives: The current study was envisaged to evaluate the effect of protocatechuic acid rich fraction of TP on type II collagen induced rheumatoid arthritis, inflammation and microbial population of gut by using rodent model.

Methods: Hydroethanolic extract of TP was fractionated with three different solvents (ethyl acetate, chloroform and n-butanol) and quantitatively analyzed with HPLC-DAD method which revealed n-butanol fraction of TP (BFTP) is rich in protocatechuic acid along with other phenolic compounds. Animals were randomly divided into five groups. Four groups

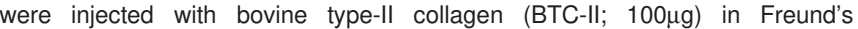
complete adjuvant $(0.1 \mathrm{ml})$ intradermally. On day 21 , animals were again treated with BTC-II along with booster incomplete Freund's adjuvant injection. After $24 \mathrm{hrs}$, animal groups were administered intraperitoneally with their respective drug treatment, i.e., vehicle, BFTP $(100,200$ and 400 $\mathrm{mg} / \mathrm{kg}$ b.w.) and methotrexate (3 $\mathrm{mg} / \mathrm{kg}$ b.w.), respectively once in a day for next 21 days. At the end of experiment serum level of pro-inflammatory cytokines was determined, and gut microbioata was analyzed by using Illumina HiSeq.

Results: Results revealed BFTP preadministered group exhibited significantly reduced level of paw swelling and arthritis score as compared to vehicle group. Inflammatory markers (IL-1 $\beta$, IL-6, IL-7, IL-23 and TNF- $\alpha$ ) of rheumatoid arthritis were also significantly alleviated by BFTP. Histological studies of mice ankle soft tissue also support the protective effect of BFTP. Additionally, BFTP repair altered gut microbial communities by reducing relative loads of inflammatory microbes such as Mucispirillum, Helicobacter and Lachnospiraceae.

Conclusion: The results conclude that BFTP possess beneficial antiinflammatory response in arthritic model and can be used as a potential anti-arthritic agent for the management of arthiritis.

Disclosure of Interests: None declared

DOI: 10.1136/annrheumdis-2019-eular.2530

\section{Rheumatoid arthritis - prognosis, predictors and outcome}

\section{SAT0070 PROTEOMIC SPECTRUM PROFILE IN INFLAMMATORY} AND DEGENERATIVE ARTHRITIS

Abeer Abdelati ${ }^{1}$, Rasha Ghazala ${ }^{2}$, Rehab Elnemr ${ }^{3}$, Fatema Dweedar ${ }^{2}$, Reham Shams-Eldeen ${ }^{2}$, Noha Kandeel ${ }^{4} .^{1}$ Department of Internal Medicine, Rheumatology Unit, Faculty of Medicine, Alexandria University., Alexandria, Egypt, ${ }^{2}$ Department of Medical Biochemistry, Faculty of Medicine, Alexandria University., Alexandria, Egypt, ${ }^{3}$ Department of Physical Medicine, Rheumatology and Rehabilitation, Faculty of Medicine, Alexandria University., Alexandria, Egypt, ${ }^{4}$ Department of Chemical Pathology, Medical Research Institute, Alexandria University, Alexandria, Egypt

Background: The identification and quantification of protein biomarkers present in tissues and body fluids is a new area of interest in the clinical management of rheumatic diseases. Proteomics may identify novel biomarker(s) which assist in understanding the pathogenesis of rheumatic disorders with diagnostic and prognostic implications. In addition, proteomic techniques in RA may be helpful to identify newer autoantibodies and inflammatory acute phase proteins.

Yet, the integration of potential biomarkers resulting from rheumatic proteomics analyses in differential diagnosis of rheumatologic disorders is not yet fully established and so several studies are needed to confirm the efficacy of these approaches.

Objectives: The purpose of this study was to use Matrix Assisted Laser Desorption lonization - Time of Flight - Mass Spectrometry (MALDI-TOFMS) to identify differentially expressed disease-related and condition-specific peptide in serum of patients with Rheumatoid arthritis (RA) and osteoarthritis (OA).

Methods: Hundred participants were divided into three groups as follow ing: RA group (35 patients with Rheumatoid arthritis representing patients with inflammatory arthritis); OA group (35 patients with Osteoarthritis representing patients with non-inflammatory arthritis); healthy control group (30 healthy volunteers). All participants were subjected to full history taking; clinical examination; and clinical Assessment of disease activity in RA patients using Disease activity score 28 (DAS-28); routine laboratory investigations; Acute phase reactants; serological tests; plain $x$-ray of affected joints, as well as serum proteomic profile using Magnetic beads (MB) separation and (MALDI - TOF - MS). ${ }^{[1]}$ Using the spectral data from the three groups, three different classification models for the three groups were generated using GA, SNN and QC algorithms. The GA model showed the best sensitivity and specificity in the three trials (RA versus control, $\mathrm{OA}$ versus control, and RA versus $\mathrm{OA}$ ).

Results: In comparing RA versus control group; the results revealed 101 peaks that discriminated the RA from the control group; 53 were significant (PWKW p>0.05) and 48 nonsignificant. Three peaks $(14 ; 25 ; 62)$ were significant and 2 were nonsignificant $(43 ; 53)$.

Regarding OA versus control group; 95 peaks were identified that discriminated the OA from the control group; 38 were significant (PWKW $p>0.05)$ and 57 nonsignificant. Three peaks $(18 ; 3 ; 14)$ were significant and peak 75 and 23 were nonsignificant.

Finally the RA group was discriminated from the OA group, the trial showed 113 peaks 73 were significant (PWKW p>0.05) and 40 nonsignificant. Five peaks were integrated (75:7767.82 $\quad 40: 2953.29 \quad 41: 2991.59$ 48:4054.75 68:6434.51), and all integrated peaks were significant. The integrated peaks showed that all peaks were downregulated except peak 40 was upregulated.

Conclusion: Data obtained from proteomic analysis allow differentiation between RA, OA, and healthy subjects. Because of its simplicity and accuracy, (MALDI-TOF-MS) is a promising method for identifying differentially expressed inflammatory versus degenerative disease-related peptide.

\section{REFERENCE}

[1] Lorenz P, Ruschpler P, Koczan D, Stiehl P, Thiesen HJ. From transcriptome to proteome: differentially expressed proteins identified in synovial tissue of patients suffering from rheumatoid arthritis and osteoarthritis by 